TRANSACTIONS OF THE

AMERICAN MATHEMATICAL SOCIETY

Volume 355, Number 3, Pages 1183-1199

S 0002-9947(02)03165-3

Article electronically published on November 7, 2002

\title{
THE MORI CONES OF MODULI SPACES OF POINTED CURVES OF SMALL GENUS
}

\author{
GAVRIL FARKAS AND ANGELA GIBNEY
}

\begin{abstract}
We compute the Mori cones of the moduli spaces $\bar{M}_{g, n}$ of $n$ pointed stable curves of genus $g$, when $g$ and $n$ are relatively small. For instance we show that for $g<14$ every curve in $\bar{M}_{g}$ is equivalent to an effective combination of the components of the locus of curves with $3 g-4$ nodes. We completely describe the cone of nef divisors for the space $\bar{M}_{0,6}$, thus verifying Fulton's conjecture for this space. Using this description we obtain a classification of all the fibrations of $\bar{M}_{0,6}$.
\end{abstract}

\section{INTRODUCTION}

In this paper we describe the Mori cone of curves of the moduli space $\bar{M}_{g, n}$ of $n$-pointed stable curves of small genus $g$. Although important aspects of the birational geometry of $\bar{M}_{g, n}$ heavily depend on whether $g$ is large with respect to $n$ (e.g., the Kodaira dimension), it turns out that surprisingly the problem of determining the cone of curves $\overline{N E}_{1}\left(\bar{M}_{g, n}\right)$ can be expressed entirely in terms of the geometry of the moduli spaces $\bar{M}_{0, g+n}$ of rational curves with marked points (cf. [GKM]).

There is a combinatorial stratification of $\bar{M}_{g, n}$ given by topological type and the components of the 1-dimensional stratum, that is, loci of curves with $(3 g-4+n)$ nodes are called Faber curves (or $F$-curves). Our first result is that any curve in $\bar{M}_{g, n}$ is numerically equivalent to an effective combination of these $F$-curves, at least when the genus is relatively small:

Theorem 1. The Mori cone of curves $\overline{N E}_{1}\left(\bar{M}_{g, n}\right)$ is generated by F-curves when $g \leq 13, n=0$ or $g \leq 8, n=1$ or $g=6, n=2$.

Thus in this range the cone of nef divisors is described by a simple set of inequalities corresponding to the numerical properties of all $F$-curves (cf. GKM]). This result was known when $n=0$ for $g \leq 11$ (cf. [KMcK]), and when $n=1, g \leq 6$ (cf. [GKM]). The case $n=0, g \leq 4$ was first settled by Faber (cf. [Fa1]).

Already for small $n$ the moduli spaces $\bar{M}_{0, n}$ are quite intricate objects deeply rooted in classical algebraic geometry. For instance, $\bar{M}_{0,5}$ is a del Pezzo surface of degree 5 while $\bar{M}_{0,6}$ is a small resolution of two famous modular varieties: the Segre cubic $\mathcal{S}_{3} \subseteq \mathbb{P}^{4}$, which is the symmetric GIT moduli space of 6 points on $\mathbb{P}^{1}$ and is the unique cubic threefold with 10 nodes, and its dual, the Igusa quartic $\mathcal{I}_{4} \subseteq \mathbb{P}^{4}$,

Received by the editors February 25, 2002.

2000 Mathematics Subject Classification. Primary 14H10.

(C)2002 American Mathematical Society 
which is the GIT moduli space of 6 points on $\mathbb{P}^{2}$ which lie on a conic and whose singular locus consists of 15 double lines (cf. $[\mathrm{H}]$ ).

Since the space $\bar{M}_{0, n}$ has a combinatorial description somewhat similar to that of a toric variety (although $\bar{M}_{0, n}$ itself is certainly not a toric variety), Fulton asked whether any effective (nef) divisor on $\bar{M}_{0, n}$ is linearly equivalent to an effective combination of boundary divisors corresponding to singular curves. We prove the following result:

Theorem 2. The cone of nef divisors of $\bar{M}_{0,6}$ is contained in the convex hull of boundary classes, and it has a natural decomposition into 11 subcones.

The precise inequalities defining these subcones can be found in Section 4. The explicit decomposition of the nef cone of $\bar{M}_{0,6}$ enables us to classify all fibrations of $\bar{M}_{0,6}$. Recall that a morphism $f: X \rightarrow Y$ with $X$ and $Y$ being irreducible projective varieties is said to be a fibration if $\operatorname{dim}(X)>\operatorname{dim}(Y)$ and $f_{*} \mathcal{O}_{X}=\mathcal{O}_{Y}$, that is, $f$ is its own Stein factorization. We have the following:

Theorem 3. 1. Any fibration of $\bar{M}_{0,5}$ factors through a projection $\bar{M}_{0,5} \rightarrow \bar{M}_{0,4}$ dropping one of the marked points.

2. Any fibration of $\bar{M}_{0,6}$ factors through a projection $\bar{M}_{0,6} \rightarrow \bar{M}_{0, n}, n \in\{4,5\}$, dropping one or two points, or through a projection $\bar{M}_{0,6} \rightarrow \bar{M}_{0,4} \times \bar{M}_{0,4}$ obtained by dropping two disjoint pairs of marked points.

The fact that every nef divisor on $\bar{M}_{0,6}$ is linearly equivalent to an effective combination of boundary divisors (that is, the first part of Theorem 2) has been previously checked by Faber (cf. [Fa2]) and by Keel (using the computer program Porta). The salient features of our Theorem 2 are the method of proof, which can be applied in more general situations (see Propositions 8 and 9), and the decomposition of the nef cone of $\bar{M}_{0,6}$ into subcones, which in particular leads to a classification of all fibrations of $\bar{M}_{0,6}$. For example, the fibrations $\bar{M}_{0,6} \rightarrow \bar{M}_{0,4} \times \bar{M}_{0,4}$ correspond to nef divisors lying in the boundary of two different chambers (see Section 4 for a precise statement). The main idea in the proof of Theorem 2 is to write every divisor $D$ on $\bar{M}_{0,6}$ canonically as a combination of boundary classes with coefficients being intersection numbers of $D$ with precisely determined $F$-curves. We hope that a similar approach could lead to a solution to Fulton's conjecture for all spaces $\bar{M}_{0, n}$.

We note that Theorem 2 gives a new combinatorial proof that $\overline{N E}_{1}\left(\bar{M}_{0,6}\right)$ is spanned by $F$-curves (cf. $\mathrm{KMcK}$, Theorem 1.2). The original proof used that $\bar{M}_{0, n}$ is a $\mathbb{Q}$-Fano variety for $n \leq 6$ (which is not the case for any $n \geq 7$ ).

Sean Keel showed that there are effective divisors on $\bar{M}_{0,6}$ which are not expressible as effective combinations of boundary classes (see also [Ve]). For example, if $\phi: \bar{M}_{0,6} \rightarrow \bar{M}_{3}$ is the map obtained by identifying three pairs of points on a 6-pointed rational curve and $\overline{\mathcal{H}} \subseteq \bar{M}_{3}$ is the locus of hyperelliptic curves, then $\phi^{*}(\overline{\mathcal{H}})$ is not linearly equivalent to an effective sum of boundary classes. Hassett and Tschinkel recently proved that the effective cone on $\bar{M}_{0,6}$ is spanned by the boundary classes and the pull-backs $\phi^{*}(\overline{\mathcal{H}})$ corresponding to all possibilities of identifying three pairs of points (cf. [HT]). In light of their work, Theorem 2 shows that the nef cone of $\bar{M}_{0,6}$ is very small with respect to the effective cone of divisors. Indeed, while we show that any nef divisor may be expressed as an effective sum of 
the 25 boundary classes, one needs another 15 divisor classes to describe all effective divisors.

Our proof of Theorem 1 makes use of the following "bridge" theorem (cf. GKM], Theorem 0.3): if $\psi: \bar{M}_{0, g+n} / S_{g} \rightarrow \bar{M}_{g, n}$ is the map given by attaching elliptic tails to the first $g$ marked points of a $(g+n)$-pointed rational curve, then a divisor $D$ on $\bar{M}_{g, n}$ is nef if and only if $\psi^{*}(D)$ is nef and $D$ meets all $F$-curves on $\bar{M}_{g, n}$ nonnegatively. In other words, to show that $\overline{N E}_{1}\left(\bar{M}_{g, n}\right)$ is generated by $F$-curves, it suffices to prove the similar statement on the space $\bar{M}_{0, g+n} / S_{g}$.

We note that Theorem 3 should be compared to Gibney's result that for $g \geq 2$ any fibration of $\bar{M}_{g, n}$ factors through a projection to some $\bar{M}_{g, i}(i<n)$ dropping some of the marked points (cf. G], GKM], Corollary 0.10). Paradoxically, because of the combinatorial complexity of $\operatorname{Pic}\left(\bar{M}_{0, n}\right)$, the fibration problem is much more difficult in genus 0 than in higher genus!

Acknowledgments: We are grateful to Igor Dolgachev, Bill Fulton and Sean Keel for many interesting discussions related to this project.

\section{Generalities on $\bar{M}_{0, n}$}

We record a few facts about the moduli space $\bar{M}_{0, n}$ of stable rational $n$-pointed curves. For more information about $\bar{M}_{0, n}$, see for example Kap or [Ke]. Throughout the paper we work exclusively with $\mathbb{Q}$-divisors, and all the Picard groups we consider are with rational coefficients.

A vital codimension- $k$-stratum is a component of the closure of the locus of points in $\bar{M}_{0, n}$ that correspond to curves with $k$ nodes. The boundary of $\bar{M}_{0, n}$ is composed of the vital codimension-1-strata $\Delta_{S}$, where $S \subset\{1, \ldots, n\}$ with $|S|,\left|S^{c}\right| \geq 2$. We always have the identification $\Delta_{S}=\Delta_{S^{c}}$. We denote by $\delta_{S}$ the linear equivalence class of $\Delta_{S}$ in Pic $\left(\bar{M}_{0, n}\right)$. An effective 1-cycle that is numerically equivalent to a vital 1-stratum is also known as an F-curve. By an F-divisor we mean a divisor that nonnegatively intersects the $F$-curves.

We will consider the tautological classes $\psi_{i}=c_{1}\left(\mathbb{L}_{i}\right)$ for $1 \leq i \leq n$, where $\mathbb{L}_{i}$ is the line bundle on $\bar{M}_{0, n}$ whose fibre over the moduli point $\left[C, x_{1}, \ldots, x_{n}\right]$ is $T_{x_{i}}^{\vee}(C)$. Recall also that there exists an ample divisor class

$$
\kappa_{1}=\sum_{\substack{S \subset\{1, \ldots, n\} \\|S| \leq n / 2}} \frac{(|S|-1)(n-|S|-1)}{(n-1)} \delta_{S}
$$

whose support is the whole boundary of $\bar{M}_{0, n}$ (cf. [AC]).

For each subset $S \subset\{1, \ldots, n\}$ we denote by $G_{S}$ the stabilizer of $\delta_{S}$ under the natural action of $S_{n}$ on $\bar{M}_{0, n}$. Then the $G_{S}$-invariant divisor classes of the form

$$
\delta_{b}^{S, a}:=\sum_{\substack{A \subset S,|A|=a \\ B \subset S^{c},|B|=b}} \delta_{A \cup B}
$$

generate $\operatorname{Pic}\left(\bar{M}_{0, n}\right)^{G_{S}}$. We have the following relation between tautological and boundary classes: 
Lemma 1. The tautological classes $\psi_{i}$, for $1 \leq i \leq n$, have the following average expression in terms of $G_{\{i\}}$-invariant boundaries:

$$
\psi_{i} \equiv \sum_{j=1}^{n-3} \frac{(n-1-j)(n-2-j)}{(n-1)(n-2)} \delta_{j}^{\{i\}, 1} .
$$

Proof. We use that, given two distinct elements $q, r \in\{1, \ldots, n\}-\{i\}$, we have that $\psi_{i} \equiv \sum_{\substack{i \in S \\ q, r \notin S}} \delta_{S}$ (cf. [AC], Proposition 1.6). We then average all such relations obtained by varying $q$ and $r$.

The following average relation between $G_{S^{-}}$-invariant divisor classes will be used throughout the paper:

Proposition 1. Suppose $S \subset\{1, \ldots, n\}$ has s elements. The following relation in $\operatorname{Pic}\left(\bar{M}_{0, \mathrm{n}}\right)$ holds:

$$
\delta_{S}=\sum_{\substack{1 \leq a \leq s,(a, b) \neq(s, 0) \\ 0 \leq b \leq n-s-1}} \eta_{s, a, b} \delta_{b}^{S, a},
$$

where

$$
\eta_{s, a, b}:=\frac{a(b+s-n)(1+b+a(n-1)-n+s-s(a+b))}{s(s-1)(n-s)(n-s-1)} .
$$

Proof. We use Keel's relation in $\operatorname{Pic}\left(\bar{M}_{0, n}\right)$ (cf. [Ke] $)$ : given four distinct elements $p, q, r, s \in\{1, \ldots, n\}$, we have that

$$
\sum_{\substack{p, q \in T \\ r, s \notin T}} \delta_{T} \equiv \sum_{\substack{p, r \in T \\ q, s \notin T}} \delta_{T} \equiv \sum_{\substack{p, s \in T \\ q, r \notin T}} \delta_{T}
$$

Having fixed $S$, we write down all possible such relations for which $p, q \in S$ and $r, s \notin S$. Then we add them together and average.

It is well known that the boundary classes $\delta_{S}$ generate $\operatorname{Pic}\left(\bar{M}_{0, n}\right)$ (cf. $\left.\overline{\mathrm{Ke}}\right]$ ). The existence of many relations between the $\delta_{S}$ 's, hence the absence of a "canonical" basis of $\operatorname{Pic}\left(\bar{M}_{0, n}\right)$, reflects the combinatorial complexity of $\bar{M}_{0, n}$. Using Kapranov's description of $\bar{M}_{0, n}$ as the space obtained from $\mathbb{P}^{n-3}$ after a sequence of $n-4$ blow-ups, one sees that $\psi_{n}$ and the boundaries $\delta_{S \cup\{n\}}$ with $S \subset\{1, \ldots, n-1\}$ and $|S| \leq n-4$ constitute a basis for $\operatorname{Pic}\left(\bar{M}_{0, n}\right)$. In particular, $\rho\left(\bar{M}_{0, n}\right)=2^{n-1}-\left(\begin{array}{l}n \\ 2\end{array}\right)-1$. However, this basis singles out the $n$-th marked points, and we chose to express all our calculations in a basis which treats all marked points equally:

Lemma 2. For $n \geq 5$, the classes $\left\{\psi_{i}\right\}_{i=1}^{n}$ and $\delta_{S}$, where $|S|,\left|S^{c}\right| \geq 3$, form a basis of $\operatorname{Pic}\left(\bar{M}_{0, \mathrm{n}}\right)$.

Proof. We denote by $V \subset \operatorname{Pic}\left(\bar{M}_{0, n}\right)$ the subspace generated by the classes $\left\{\psi_{i}\right\}_{i=1}^{n}$ and $\left\{\delta_{S}\right\}_{|S|,\left|S^{c}\right| \geq 3}$. It is enough to show that $\delta_{x y} \in V$ for all distinct $x, y$. From Proposition 1 we obtain that

$$
(n-2)(n-3) \delta_{x y}+2 \delta_{2}^{x y, 0}-(n-3) \delta_{1}^{x y, 1} \in V .
$$

By writing the relation $\psi_{i}=\sum_{\substack{i \in T \\ x, y \notin T}} \delta_{T}$ for all $i \in\{x, y\}^{c}$ and averaging, we obtain that

$$
(n-2) \delta_{x y}+2 \delta_{2}^{x y, 0} \in V .
$$


Finally, by averaging all relations $\psi_{x}+\psi_{y}=\sum_{\substack{x \in T \\ a, b \notin T}} \delta_{T}+\sum_{\substack{y \in T \\ a, b \notin T}} \delta_{T}$ over all $a, b \in$ $\{x, y\}^{c}$ we obtain that

$$
2\left(\begin{array}{c}
n-2 \\
2
\end{array}\right) \delta_{x y}+2 \delta_{2}^{x y, 0}+\left(\begin{array}{c}
n-3 \\
2
\end{array}\right) \delta_{1}^{x y, 1} \in V .
$$

Clearly (i)-(iii) imply that $\delta_{x y} \in V$.

We will often use the following notation:

Definition 1. For a divisor $D \equiv \sum_{1 \leq i \leq n} c_{i} \psi_{i}-\sum_{|S|,\left|S^{c}\right| \geq 3} b_{S} \delta_{S}$ on $\bar{M}_{0, n}$ and for a fixed subset $T \subset\{1, \ldots, n\}$, we set

$$
I^{T}:=\sum_{t \in T} c_{t}, \quad O^{T}:=\sum_{t \notin T} c_{t}, \quad \Sigma_{i}^{T, j}:=\sum_{\substack{A \subset T,|A|=j \\ B \subset T^{c},|B|=i}} b_{A \cup B} .
$$

We also recall that $F$-curves in $\bar{M}_{0, n}$ correspond to partitions $I, J, K, L$ of $\{1$, $\ldots, n\}$ into nonempty subsets. For each such partition we have a map $\nu: \bar{M}_{0,4} \rightarrow$ $\bar{M}_{0, n}$ obtained by attaching $1+|I|, 1+|J|, 1+|K|$ and $1+|L|$-pointed rational curves at each of the four marked points. Every $F$-curve in $\bar{M}_{0, n}$ is numerically equivalent to such an image $\nu\left(\bar{M}_{0,4}\right)$ corresponding to a partition (cf. [GKM], Theorem 2.2).

\section{The Fibrations of $\bar{M}_{0,5}$}

In this section we first show that any $F$-nef divisor in $\bar{M}_{0,5}$ can be expressed as an effective sum of boundary classes. Although this result can be proved in various ways, we present it because it illustrates our technique for giving a natural presentation of any divisor in terms of boundary classes via averaging. Moreover, it enables us to classify the fibrations of $\bar{M}_{0,5}$.

For $a, b \in\{1, \ldots, 5\}$ we consider the $G_{a b}$-invariant sum of $F$-curves $C^{a b}:=$ $\sum_{i \in\{a, b\}^{c}} \Delta_{a b i}$. We show that any divisor on $\bar{M}_{0,5}$ has a canonical presentation in terms of boundary divisors.

Proposition 2. If $D$ is any divisor in $\bar{M}_{0,5}$, then

$$
D \equiv \sum_{a, b \in\{1, \ldots, 5\}} \frac{1}{6}\left(C^{a b} \cdot D\right) \delta_{a b} .
$$

In particular, any $F$-divisor is an effective sum of boundary classes.

Proof. We have seen that $\left\{\psi_{i}\right\}_{i=1}^{5}$ forms a basis for $\operatorname{Pic}\left(\bar{M}_{0,5}\right)$. Let $D \equiv \sum_{1 \leq i \leq 5} c_{i} \psi_{i}$ be any divisor on $\bar{M}_{0,5}$. Using the average formula from Lemma 1

$$
\psi_{i}=\frac{1}{2}\left(\sum_{a \neq i} \delta_{a i}\right)+\frac{1}{6}\left(\sum_{a, b \neq i} \delta_{a b}\right)
$$

we can rewrite $D$ as

$$
D \equiv \sum_{a, b \in\{1, \ldots, 5\}} \frac{1}{6}\left(3 I^{a b}+O^{a b}\right) \delta_{a b} .
$$

The coefficient of $\delta_{a b}$ in this expression is just $\frac{1}{6}\left(D \cdot C^{a b}\right)$; so the conclusion follows. 
Remark. If $D \equiv \sum_{i=1}^{5} c_{i} \psi_{i}$ is an $F$-divisor on $\bar{M}_{0,5}$, we see that $D \cdot \Delta_{a b}=O^{a b} \geq 0$ for any $a, b \in\{1, \ldots, 5\}$. Moreover, if $D \cdot C^{a b}=3 I^{a b}+O^{a b}=0$, then $c_{i}=$ $-\left(c_{a}+c_{b}\right) \geq 0$, for all $i \in\{a, b\}^{c}$.

Next we prove that a nontrivial $F$-divisor on $\bar{M}_{0,5}$ is either big or the pull-back of an ample divisor under the projection $\pi_{i}: \bar{M}_{0,5} \rightarrow \bar{M}_{0,4}$ dropping the $i$-th point.

Proof of Theorem 3, Part 1. Let $D \equiv \sum_{i=1}^{5} c_{i} \psi_{i}$ be a nontrivial $F$-divisor. We have the following possibilities:

1. $D \cdot C^{a b}>0$ for any $a, b \in\{1, \ldots, 5\}$. Then using Proposition 2 we can write $D \equiv a \kappa_{1}+$ (Effective), for some $a \in \mathbb{Q}_{>0}$, and since $\kappa_{1}$ is ample, $D$ has to be big; so it does not give rise to a fibration. Thus we may assume that $D \cdot \delta_{a b}=0$ for some $a, b \in\{1, \ldots, 5\}$, say $D \cdot C^{12}=0$. There are two possibilities:

2. $D \cdot C^{1 i}>0$ for all $i \in\{1,2\}^{c}$. Then $c_{3}=c_{4}=c_{5}=c=-\left(c_{1}+c_{2}\right)>0$. Moreover, $D \cdot C^{a b}>0$ for $a, b \in\{1,2\}^{c}$, and $D \cdot C^{2 i}>0$ for all $i \in\{1,2\}^{c}$. In this case, using Proposition 2 the divisor $D$ can be rewritten as a positive combination:

$$
D \equiv \frac{5 c+3 c_{1}+c_{2}}{6} D_{1}+\frac{5 c+c_{1}+3 c_{2}}{6} D_{2}
$$

where $D_{1}=\sum_{a, b \in\{1,2\}^{c}} \delta_{a b}+\sum_{a \neq 1,2} \delta_{1 a}$ and $D_{2}=\sum_{a, b \in\{1,2\}^{c}} \delta_{a b}+\sum_{a \neq 1,2} \delta_{2 a}$.

Since $D_{2}=\pi_{1}^{*}\left(\delta_{23}+\delta_{24}+\delta_{34}\right)$ and $D_{1}=\pi_{2}^{*}\left(\delta_{13}+\delta_{14}+\delta_{34}\right)$, it follows that $D$ is the pull-back of an ample divisor under the birational map $\left(\pi_{1}, \pi_{2}\right): \bar{M}_{0,5} \rightarrow$ $\bar{M}_{0,4} \times \bar{M}_{0,4}$; hence it is big.

3. There is an $i \in\{1,2\}^{c}$ such that $D \cdot C^{1 i}=0$, say $D \cdot C^{13}=0$. Then $c_{2}=$ $c_{3}=c_{4}=c_{5}=c>0$ and $c_{1}=-2 c$. Proposition 2 gives that $D \equiv c \sum_{a, b \neq 1} \delta_{a b}=$ $c \pi_{1}^{*}\left(\delta_{23}+\delta_{24}+\delta_{25}\right)$, which proves our contention.

\section{The NEF CONE OF $\bar{M}_{0,6}$}

In this section we prove Theorems 2 and 3 The main idea is to canonically write every divisor $D$ on $\bar{M}_{0,6}$ as a linear combination of boundary divisors with coefficients being intersection numbers with specific combinations of $F$-curves.

We first introduce a number of 1-cycles on $\bar{M}_{0,6}$. Whenever we refer to a 1-cycle as being a weighted sum of $F$-curves, we mean that we divide by the number of irreducible components making up the cycle. Let us fix distinct $a, b \in\{1, \ldots, 6\}$. By $C_{1}^{a b}$ (respectively $C_{2}^{a b}$ ) we denote the weighted sum of $F$-curves of type $(3: 1: 1: 1)$ (resp. $(2: 2: 1: 1)$ ) having both points indexed by $a$ and $b$ on the spine. By $C_{3}^{a b}$ we denote the weighted sum of $F$-curves of type $(3: 1: 1: 1)$ having neither $a$ nor $b$ on the spine, while $C_{4}^{a b}$ is the weighted sum of $F$-curves of type $(2: 2: 1: 1)$ with $a, b$ on the same tail.

For $a, b \in\{2, \ldots, 6\}$, let $C_{1}^{1 a b}$ be the weighted sum of $F$-curves of type $(2: 2$ : $1: 1)$ having exactly one of the points indexed by elements of $\{1, a, b\}$ on the spine while the remaining two points are on one of the attached tails. By $C_{2}^{1 a b}$ (resp. $\left.C_{3}^{1 a b}\right)$ we denote the weighted sum of $F$-curves of type $(3: 1: 1: 1)$ having only one of the points (resp. two of the points) indexed by elements of $\{1, a, b\}$ on the spine.

It may be of interest to note that $C_{1}^{a b}$ and $C_{2}^{a b}$ are the only $G_{a b}$-invariant $F$ curves (up to rescaling) that properly intersect $\Delta_{a b}$. Similarly $C_{1}^{1 a b}$ is the unique $G_{1 a b}$-invariant $F$-curve that properly intersects $\Delta_{1 a b}$, and $C_{2}^{1 a b}$ and $C_{3}^{1 a b}$ are the only $G_{1 a b}$-invariant curves of type $(3: 1: 1: 1)$ that do not intersect $\Delta_{1 a b}$ at all. 
Throughout this section we use the notation from Definition 1. To simplify things we set $\Sigma^{a b c}:=\Sigma_{1}^{a b c, 2}, \Sigma:=\Sigma^{a b c}+\delta_{a b c}$ and $I+O:=I^{a b c}+O^{a b c}=I^{a b}+O^{a b}$, for any $a, b, c \in\{1, \ldots, 6\}$. The following lemma describes the intersections of the previously introduced curves with any divisor.

Lemma 3. If $D \equiv \sum_{1 \leq i \leq 6} c_{i} \psi_{i}-\sum_{i j \in\{2, \ldots, 6\}} b_{1 i j} \delta_{1 i j}$ is any divisor on $\bar{M}_{0,6}$, then for distinct $a, b, c \in\{1, \ldots, 6\}$ we have that

$$
\begin{gathered}
C_{1}^{a b} \cdot D=I^{a b}+\frac{1}{4} O^{a b}+\frac{1}{4} \Sigma_{1}^{a b, 2}, \quad C_{2}^{a b} \cdot D=I^{a b}-\frac{1}{3} \Sigma_{2}^{a b, 1}, \quad C_{3}^{a b} \cdot D=\frac{3}{4} O^{a b}+\frac{1}{4} \Sigma_{1}^{a b, 2}, \\
C_{4}^{a b} \cdot D=\frac{1}{2} O^{a b}-\frac{1}{2} \Sigma_{1}^{a b, 2}, \quad C_{1}^{a b c} \cdot D=\frac{1}{3}(I+O)-b_{a b c}-\frac{1}{9} \Sigma^{a b c} \\
C_{2}^{a b c} \cdot D=\frac{1}{3} I^{a b c}+\frac{2}{3} O^{a b c}+\frac{1}{9} \Sigma^{a b c}, \quad C_{3}^{a b c} \cdot D=\frac{2}{3} I^{a b c}+\frac{1}{3} O^{a b c}+\frac{1}{9} \Sigma^{a b c}
\end{gathered}
$$

Proof. This follows from standard intersection calculations as explained in, for example, [HMO] or [Fa1].

The following sufficient criterion for a divisor on $\bar{M}_{0,6}$ to be big will prove useful a number of times:

Lemma 4. Let $\{i, j, k, l, m, n\}$ be a permutation of $\{1, \ldots, 6\}$. Then any effective sum of boundary classes supported on $\delta_{m n}, \delta_{i l}, \delta_{j l}, \delta_{k l}, \delta_{m n i}, \delta_{m n j}$ and $\delta_{m n k}$ is big. Moreover, any effective class supported on $\delta_{i l}, \delta_{j m}, \delta_{k n}$ and on all boundaries $\delta_{a b c}$ except $\delta_{i j k}$ is big as well.

Proof. For the first statement it is enough to consider the pull-back of the ample class $\left(\delta_{m n}, \delta_{m n}, \delta_{m n}\right)$ under the birational map $\left(\pi_{i j}, \pi_{j k}, \pi_{i k}\right): \bar{M}_{0,6} \rightarrow \bar{M}_{0,4} \times$ $\bar{M}_{0,4} \times \bar{M}_{0,4}$ whose components forget the marked points $(i, j),(j, k)$ and $(i, k)$ respectively. To prove the second statement we pull back the class $\left(\delta_{j m}+\delta_{k n}, \delta_{i l}+\right.$ $\left.\delta_{k n}, \delta_{i l}+\delta_{j m}\right)$ via the birational map $\left(\pi_{i l}, \pi_{j m}, \pi_{k n}\right): \bar{M}_{0,6} \rightarrow \bar{M}_{0,4} \times \bar{M}_{0,4} \times$ $\bar{M}_{0,4}$.

We have the following canonical presentation of any divisor class on $\bar{M}_{0,6}$ :

Proposition 3. Any divisor $D$ on $\bar{M}_{0,6}$ can be written as

$$
\begin{aligned}
D & \equiv \sum_{a, b \in\{1, \ldots, 6\}}\left(\frac{2}{5}\left(C_{1}^{a b} \cdot D\right)+\frac{1}{5}\left(C_{2}^{a b} \cdot D\right)\right) \delta_{a b} \\
& +\sum_{a, b \in\{2, \ldots, 6\}}\left(\frac{7}{10}\left(C_{1}^{1 a b} \cdot D\right)+\frac{1}{15}\left(\left(C_{2}^{1 a b}+C_{3}^{1 a b}\right) \cdot D\right)+\frac{4}{135} \Sigma^{1 a b}\right) \delta_{1 a b} .
\end{aligned}
$$

Proof. We perform two canonical modifications of the expression of any divisor on $\bar{M}_{0,6}$ in the basis referred to in Lemma 2 In this way we get two presentations for any divisor on $\bar{M}_{0,6}$. The expression from Proposition 3 is obtained by taking a suitable linear combination of them. Note that, if $D$ is an $F$-divisor, the $\delta_{a b}$ part of the expression of $D$ is always effective.

We start with a divisor $D \equiv \sum_{1 \leq i \leq 6} c_{i} \psi_{i}-\sum_{j, k \in\{2, \ldots, 6\}} b_{1 j k} \delta_{1 j k}$. We replace each $\psi_{i}$ by its average expansion provided by Lemma 1 to get that $D$ is linearly 
equivalent to

$$
\begin{aligned}
D^{I}= & \sum_{j, k \in\{2, \ldots, 6\}}\left(\frac{3}{10}(I+O)-b_{1 j k}\right) \delta_{1 j k} \\
& \left.+\sum_{a, b \in\{1, \ldots, 6\}}\left(\frac{3}{5} I^{a b}+\frac{1}{10} O^{a b}\right)\right) \delta_{a b} .
\end{aligned}
$$

Next, in $D^{I}$ we replace each class $\delta_{1 j k}$ by its average formula from Proposition 1

$$
\delta_{1 j k}=\frac{2}{9} \sum_{\substack{a \in\{1, j, k\} \\ b \in\{1, j, k\}^{c}}} \delta_{a b}-\frac{1}{3} \sum_{\substack{a b \in\{1, j, k\} \text { or } \\ a b \in\{1, j, k\}^{c}}} \delta_{a b}+\frac{1}{9} \sum_{a, b \neq j, k} \delta_{1 a b},
$$

to get that $D$ can also be written as

$$
\begin{aligned}
D^{I I}= & \sum_{j, k \in\{2, \ldots, 6\}}\left(\frac{3}{10}(I+O)-\frac{1}{9} \Sigma^{1 j k}\right) \delta_{1 j k} \\
& +\sum_{a, b \in\{1, \ldots, 6\}}\left(\frac{3}{5} I^{a b}+\frac{1}{10} O^{a b}+\frac{1}{3} \Sigma_{1}^{a b, 2}-\frac{2}{9} \Sigma_{2}^{a b, 1}\right) \delta_{a b} .
\end{aligned}
$$

We now write that $D \equiv \frac{3}{10}\left(\frac{7}{3} D^{I}+D^{I I}\right)$, and by using the intersection numbers computed in Lemma 3 we get exactly the desired expression for $D$.

To simplify notation we shall rewrite the expression from Proposition 3 as

$$
\frac{10}{3} D=\frac{7}{3} D^{I}+D^{I I}=\sum_{a, b \in\{1, \ldots, 6\}} \zeta_{a b} \delta_{a b}+\sum_{a, b \in\{2, \ldots, 6\}} \zeta_{1 a b} \delta_{1 a b} .
$$

Thus $\zeta_{a b}=2 I^{a b}+\frac{1}{3} O^{a b}+\frac{1}{3} \Sigma_{1}^{a b, 2}-\frac{2}{9} \Sigma_{2}^{a b, 1}$ and $\zeta_{1 a b}=I+O-\frac{20}{9} b_{1 a b}-\frac{1}{9} \Sigma$. We have already seen that for an $F$-divisor all the coefficients $\zeta_{a b}$ are $\geq 0$. Moreover, in Proposition 4 we prove that at most one of the coefficients $\zeta_{1 i j}$ can be $<0$. If this happens, we replace $\delta_{1 i j}$ by its average expression (1), spreading the negativity of $\zeta_{1 i j}$ among all boundary classes. We show that the resulting expression becomes effective, thus proving Theorem 2. This procedure gives a decomposition of the nef cone of $\bar{M}_{0,6}$ into 11 natural subcones: one described by inequalities $\zeta_{1 a b} \geq 0$ for all $a, b \in\{2, \ldots, 6\}$, and the remaining 10 given by inequalities $\zeta_{1 i j} \leq 0$ for $i, j \in\{2, \ldots, 6\}$. More precisely, we have the following result:

Proposition 4. Let $D$ be an F-divisor on $\bar{M}_{0,6}$ with $\zeta_{1 i j}<0$ for some $i, j \in$ $\{2, \ldots, 6\}$. Then $D$ is big, and there exists a big effective combination of boundary classes $B_{1 i j}$ such that

$$
\begin{aligned}
D & \equiv B_{1 i j}+\sum_{\substack{a \in\{1, i, j\} \\
b \in\{1, i, j\}^{c}}}\left(\frac{1}{6}\left(C_{1}^{1 i j} \cdot D\right)+\frac{2}{3}\left(C_{1}^{a b} \cdot D\right)\right) \delta_{a b} \\
+ & \sum_{\substack{a, b \in\{1, i, j\} \text { or } \\
a, b \in\{1, i, j\}^{c}}}\left(\frac{2}{5}\left(C_{1}^{a b} \cdot D\right)+\frac{1}{5}\left(C_{2}^{a b} \cdot D\right)\right) \delta_{a b}+\sum_{a, b \neq i, j} \frac{2}{3}\left(C_{a b} \cdot D\right) \delta_{1 a b},
\end{aligned}
$$

where $C_{a b}$ is an effective sum of $F$-curves. In particular, Theorem 0 follows. 
Proof. After replacing $\delta_{1 i j}$ by its average expression in (2) we obtain the identity $(*)$

$$
\begin{aligned}
\frac{10}{3} D \equiv & \sum_{\substack{a \in\{1, i, j\} \\
b \in\{1, i, j\}^{c}}}\left(\zeta_{a b}+\frac{2}{9} \zeta_{1 i j}\right) \delta_{a b}+\sum_{\substack{a, b \in\{1, i, j\} \text { or } \\
a, b \in\{1, i, j\}\}^{c}}}\left(\zeta_{a b}-\frac{1}{3} \zeta_{1 i j}\right) \delta_{a b} \\
& +\sum_{a, b \neq i, j}\left(\zeta_{1 a b}+\frac{1}{9} \zeta_{1 i j}\right) \delta_{1 a b .} .
\end{aligned}
$$

We set $\rho:=-(I+O+\Sigma)$. Since $0>\zeta_{1 i j} \geq \zeta_{1 i j}-\frac{5}{2} C_{1}^{1 i j} \cdot D=-\frac{1}{6} \rho$, we obtain that $\rho>0$. It is rather straightforward to check using Lemma 3 that

$$
\begin{gathered}
\zeta_{a b}+\frac{2}{9} \zeta_{1 i j}=\frac{5}{9} C_{1}^{1 i j} \cdot D+\frac{20}{9} C_{1}^{a b} \cdot D+\frac{5}{27} \rho, \text { for } a \in\{1, i, j\} \text { and } b \in\{1, i, j\}^{c}, \\
\frac{1}{9} \zeta_{1 i j}+\zeta_{1 a b}=\frac{2 \rho-8 \zeta_{1 i j}}{9}+\frac{20}{9}\left(I+O-b_{1 i j}-b_{1 a b}\right), \text { for }\{a, b\} \neq\{i, j\}
\end{gathered}
$$

while obviously $\zeta_{a b}-\frac{1}{3} \zeta_{1 i j}>0$ for $a, b \in\{1, i, j\}$ or $a, b \in\{1, i, j\}^{c}$. We claim that $(*)$ is already an effective representation of $\frac{10}{3} D$. As it turns out, we can prove a little more than that.

For $a, b \in\{2, \ldots, 6\}$ such that $\{a, b\} \neq\{i, j\}$ we define an effective 1-cycle $C_{a b}$ such that $C_{a b} \cdot D=(I+O)-b_{1 i j}-b_{1 a b}$. By passing to the complement if necessary, we may assume that $\{i, j\} \cap\{a, b\}=\emptyset$. We denote by $k$ the remaining marked point; hence $\{1, \ldots, 6\}=\{1, i, j, k, a, b\}$. We then take $C_{a b}:=\frac{1}{2}\left(2 C_{3}^{1 k}+C_{4}^{1 k}+C^{\prime}\right)$, where $C^{\prime}$ is the $F$-curve of type $(2: 2: 1: 1)$ with $i, j$ and $a, b$ respectively sitting on different tails.

We define the divisor class $B_{1 i j}$ by the formula

$$
B_{1 i j}=\frac{3}{10}\left(\frac{5 \rho}{27} \sum_{\substack{a \in\{1, i, j\} \\ b \in\{1, i, j\}^{c}}} \delta_{a b}-\frac{\zeta_{1 i j}}{3} \sum_{\substack{a, b \in\{1, i, j\} \\ a, b \in\{1, i, j\}^{c}}} \delta_{a b}+\frac{2 \rho-8 \zeta_{1 i j}}{9} \sum_{a, b \neq i, j} \delta_{1 a b}\right) .
$$

All the coefficients in this expression are positive while the support of $B_{1 i j}$ is $\sum_{S \neq\{1, i, j\}} \Delta_{S}$, which is a big divisor (use Lemma 4).

We shall use Proposition 4 to classify all fibrations of $\bar{M}_{0,6}$. We have already seen that an $F$-divisor $D$ for which there exists a coefficient $\zeta_{1 i j}<0$ has to be big; hence it does not give rise to a fibration. The divisor $D$ is also big when $\zeta_{1 a b}>0$ and $\zeta_{a b}>0$ for all $a$ and $b$ (use the existence of the ample class $\kappa_{1}$ ). So we are left with classifying nontrivial $F$-divisors $D$ for which all of the coefficients in (2) are nonnegative and at least one of them is 0 . We have three cases to consider:

(1) There are at least two coefficients $\zeta_{1 a b}$ which are equal to 0 , that is, $D$ lies in the intersection of two of the subcones making up the nef cone of $\bar{M}_{0,6}$. Then we show that $D$ is the pull-back of an effective divisor via a fibration $\bar{M}_{0,6} \rightarrow \bar{M}_{0,4} \times \bar{M}_{0,4}$ obtained by forgetting two disjoint pairs of marked points.

(2) There is an $i \in\{1, \ldots, 6\}$ such that $\zeta_{i j}=0$ for all $j \neq i$. Then $D$ is the pullback of an effective divisor via the fibration $\pi_{i}: \bar{M}_{0,6} \rightarrow \bar{M}_{0,5}$ forgetting the $i$-th marked point.

(3) If neither of the previous situations occurs, then we show that $D$ is big. 
The following observation will come into nearly every argument in the rest of this section:

Lemma 5. Let $D \equiv \sum_{i=1}^{6} c_{i} \psi_{i}-\sum_{i, j \in\{2, \ldots, 6\}} b_{1 i j} \delta_{1 i j}$ be a nontrivial $F$-divisor on $\bar{M}_{0,6}$, and $\{a, b, i, j, m, n\}$ a permutation of $\{1, \ldots, 6\}$. If $\zeta_{i j}=\zeta_{a b}=0$, then $\zeta_{m n}>0$. Moreover, if $\zeta_{i a}=\zeta_{i b}=\zeta_{i m}=\zeta_{i n}=0$, then $\zeta_{i j}=0$ as well.

Proof. Without loss of generality we may assume that $\zeta_{12}=\zeta_{34}=\zeta_{56}=0$, and we prove that in this case $D$ is trivial. Our assumption implies that $C_{1}^{12} \cdot D=0$, from which we can write that $c_{i}+b_{12 i}=-\left(c_{1}+c_{2}\right)$, for all $i \in\{1,2\}^{c}$. Similarly $c_{j}+b_{34 j}=-\left(c_{3}+c_{4}\right)$, for all $j \in\{3,4\}^{c}$, and $c_{k}+b_{56 k}=-\left(c_{5}+c_{6}\right)$ when $k \in\{5,6\}^{c}$. It is easy to see that these relations imply that all the $c_{i}$ 's are equal, that is, $c_{i}=c$ for $i \in\{1, \ldots, 6\}$, and hence $b_{12 i}=-3 c$, for each $i \in\{1,2\}^{c}$. Similarly $b_{34 j}=-3 c$ for $j \in\{3,4\}^{c}$, and $b_{56 k}=-3 c$ for each $k \in\{5,6\}^{c}$.

On the other hand, $C_{2}^{12} \cdot D=0$, which implies in particular that $c_{1}+c_{2}-b_{134}-$ $b_{156}=0$, thus giving that $c_{i}=0$ for all $i \in\{1, \ldots, 6\}$. It immediately follows that the boundary coefficients must vanish too, and hence $D$ is trivial.

For the second part, let us assume that $\zeta_{12}=\cdots=\zeta_{15}=0$ and prove that $\zeta_{16}=0$. Since $C_{1}^{1 i} \cdot D=0$ for all $i \in\{2, \ldots, 5\}$, we have that $b_{1 i 6}=-I^{1 i 6}$ for all $i \in\{2, \ldots, 5\}$, which yields $C_{1}^{16} \cdot D=0$. We also know that $C_{2}^{1 i} \cdot D=0$, which turns out to be equivalent with $2 c_{1}+I+O=0$. It follows that $C_{2}^{16} \cdot D=0$ as well; hence $\zeta_{16}=0$.

We proceed with the classification of all $F$-divisors on $\bar{M}_{0,6}$. The next lemmas deal with the first two situations:

Lemma 6. Let $D \equiv \sum_{i=1}^{6} c_{i} \psi_{i}-\sum_{i, j \in\{2, \ldots, 6\}} b_{1 i j} \delta_{1 i j}$ be an F-divisor on $\bar{M}_{0,6}$ such that two coefficients $\zeta_{1 i j}$ vanish, say $\zeta_{1 i j}=\zeta_{1 k l}=0$, where $\{1, i, j, k, l, m\}$ is a permutation of $\{1, \ldots, 6\}$. Then $D$ is the pull-back of an effective divisor via the fibration $\phi=\left(\pi_{i j}, \pi_{k l}\right): \bar{M}_{0,6} \rightarrow \bar{M}_{0,4} \times \bar{M}_{0,4}$ whose components forget the marked points labelled $(i, j)$ and $(k, l)$ respectively.

Proof. We use the notation from the proof of Proposition 4 . Without loss of generality we may assume that $\zeta_{123}=\zeta_{145}=0$. From (4) we obtain that $\rho=-(I+O+\Sigma)=$ 0 and that $C_{16} \cdot D=0$, which implies that $C_{3}^{16} \cdot D=C_{4}^{16} \cdot D=0$. Since $\zeta_{123}=0$, we can also write that $\rho / 15=C_{1}^{123} \cdot D=0$ and similarly $C_{1}^{145} \cdot D=0$. Thus the intersection numbers of $D$ with every component of $C_{3}^{16}, C_{4}^{16}, C_{1}^{123}$ and $C_{1}^{145}$ respectively has to be 0 . This gives rise to 28 relations between the coefficients $c_{i}$ and $b_{1 i j}$. By writing out these relations it turns out that (2) can be rewritten as

$$
\begin{aligned}
D \equiv & (\alpha+\beta)\left(\delta_{124}+\delta_{134}+\delta_{125}+\delta_{135}\right) \\
& +\alpha\left(\delta_{12}+\delta_{13}+\delta_{62}+\delta_{63}+\delta_{146}+\delta_{156}+\delta_{16}+\delta_{23}\right) \\
& +\beta\left(\delta_{14}+\delta_{15}+\delta_{64}+\delta_{65}+\delta_{126}+\delta_{136}+\delta_{16}+\delta_{45}\right)
\end{aligned}
$$

where $\alpha=\frac{2}{3}\left(c_{1}+c_{2}\right) \geq 0$ and $\beta=\frac{2}{3}\left(c_{1}-c_{2}\right) \geq 0$. In order to finish the proof it is enough to notice that $D \equiv \alpha \pi_{45}^{*}\left(\delta_{12}+\delta_{23}+\delta_{13}\right)+\beta \pi_{23}^{*}\left(\delta_{14}+\delta_{45}+\delta_{15}\right)$.

Remark. Lemma [6] also shows that if $D$ is a nontrivial $F$-divisor on $\bar{M}_{0,6}$, then at most two of the coefficients $\zeta_{1 a b}$ can vanish.

Lemma 7. Let $D$ be an $F$-divisor such that there exists $i \in\{1, \ldots, 6\}$ with $\zeta_{i j}=0$ for all $j \neq i$. Then $D$ is the pull-back of an effective divisor under the projection $\pi_{i}: \bar{M}_{0,6} \rightarrow \bar{M}_{0,5}$ dropping the $i$-th marked point. 
Proof. Clearly we can assume that $i=1$. The hypothesis $\zeta_{1 j}=0$ for $j \in\{2, \ldots, 6\}$ is equivalent to $C_{1}^{1 j} \cdot D=C_{2}^{1 j} \cdot D=0$ for all $j \in\{2, \ldots, 6\}$. This gives that $b_{1 i j}=-I^{1 i j}$ for all $i, j \in\{2, \ldots, 6\}$. It also follows that $2 c_{1}+I+O=0$ and $I+O+\Sigma=0$. Then (2) reads

$$
D \equiv \sum_{i, j \in\{2, \ldots, 6\}} \frac{2}{3} I^{i j}\left(\delta_{i j}+\delta_{1 i j}\right)=\pi_{1}^{*}\left(\sum_{i, j \in\{2, \ldots, 6\}} \frac{2}{3} I^{i j} \delta_{i j}\right) .
$$

Proof of Theorem 3 Part 2. We start with a nontrivial $F$-divisor $D$ on $\bar{M}_{0,6}$ with $\zeta_{1 a b} \geq 0$ for all $a, b \in\{2, \ldots, 6\}$ and such that at most one coefficient $\zeta_{1 a b}$ is equal to 0 , say $\zeta_{123}=0$. Moreover, we can assume that for each $i$ there is a $j \neq i$ such that $\zeta_{i j} \neq 0$. Then we show that $D$ is big. Note that the case $\zeta_{1 a b}>0$ for all $a, b \in\{2, \ldots, 6\}$ is similar (and simpler).

Lemma 5 limits the number of coefficients $\zeta_{i j}$ that can vanish, and a case by case analysis shows that we can always find sufficiently many boundaries $\delta_{i j}$ on which $D$ is supported. Then we apply Lemma 4 to conclude that $D$ is big.

\section{The Mori COne of $\bar{M}_{g}$}

In this section we show that $\overline{N E}_{1}\left(\bar{M}_{g}\right)$ is spanned by $F$-curves for all $g \leq 13$. To prove this, it is enough to show that every $S_{g}$-invariant extremal ray on $\bar{M}_{0, g}$ is generated by an $F$-curve (cf. GKM], Theorem 0.3 ). We achieve this inductively by writing every nontrivial $S_{g}$-invariant nef divisor on $\bar{M}_{0, g}$ as a sum $K_{\bar{M}_{0, g}}+\sum_{S} a_{S} \delta_{S}$, where $0 \leq a_{S} \leq 1$ for all $S$. We also notice that for any $g \geq 14$ there are $S_{g}$-invariant $F$-divisors on $\bar{M}_{0, g}$ not of this form, thus hinting that the nature of $\bar{M}_{g}$ changes in a subtle way when $g=14$. Finally we present a combinatorial set-up enabling us to compute Mori cones of moduli spaces of 1- and 2-pointed curves of genus $g \leq 8$. It is clear that in the same way at least a couple of other cases can be settled as well.

We start by setting some notation. We denote $\widetilde{M}_{0, n}:=\bar{M}_{0, n} / S_{n}$ and we identify divisors on $\widetilde{M}_{0, n}$ with $S_{n}$-invariant divisors on $\bar{M}_{0, n}$. The spaces $\widetilde{M}_{0, n}$ are interesting for their own sake. For instance, $\widetilde{M}_{0,2 g+2}$ is isomorphic to the closure in $\bar{M}_{g}$ of the locus of hyperelliptic curves of genus $g$. For $2 \leq i<\lfloor n / 2\rfloor$, we set $B_{i}:=\sum_{\substack{S \subset\{1, \ldots, g\} \\|S|=i}} \delta_{S}$. When $i=n / 2$ we define $B_{i}:=\sum_{\substack{S \subset\{1, \ldots, g\} \\|S|=i, 1 \in S}} \delta_{S}$.

Keel and McKernan proved the following results about the Mori theory of $\widetilde{M}_{0, n}$ (cf. [KMcK], Theorem 1.3):

Proposition 5. (1) The effective cone $\overline{N E}^{1}\left(\widetilde{M}_{0, n}\right)$ is generated by the classes of the divisors $B_{i}$ for $2 \leq i \leq\lfloor n / 2\rfloor$. Any nontrivial nef divisor on $\widetilde{M}_{0, n}$ is big.

(2) For $n \leq 7$ the cone of curves $\overline{N E}_{1}\left(\bar{M}_{0, n}\right)$ is generated by $F$-curves. We also have that for $n \leq 11$ the cone $\overline{N E}_{1}\left(\widetilde{M}_{0, n}\right)$ is spanned by $F$-curves.

Remark. The previous result combined with Theorem 0.3 from GKM gives that $\overline{N E}_{1}\left(\bar{M}_{g, n}\right)$ is spanned by $F$-curves whenever $g+n \leq 7$. We also obtain that for $g \leq 11$ every $F$-divisor on $\bar{M}_{g}$ is nef. We shall extend this result for all $g \leq 13$. 
We recall that for any $S \subset\{1, \ldots, n\}$ such that $|S|,\left|S^{c}\right| \geq 2$, there is an isomorphism

$$
\phi: \bar{M}_{0,|S|+1} \times \bar{M}_{0,\left|S^{c}\right|+1} \longrightarrow \Delta_{S} \subseteq \bar{M}_{0, n}
$$

given by attaching a rational $(|S|+1)$-pointed curve to a rational $\left(\left|S^{c}\right|+1\right)$-pointed curve at a point $x$. It turns out that $\phi$ induces an isomorphism between Mori cones $\overline{N E}_{1}\left(\Delta_{S}\right)=\overline{N E}_{1}\left(\bar{M}_{0,|S|+1}\right) \times \overline{N E}_{1}\left(\bar{M}_{0, \mid S^{c \mid+1}}\right)$ (cf. KMcK, Lemma 3.8). Moreover, if $\pi_{1}: \Delta_{S} \rightarrow \bar{M}_{0,|S|+1}$ and $\pi_{2}: \Delta_{S} \rightarrow \bar{M}_{0,\left|S^{c}\right|+1}$ are the two projections, then

$$
N_{\Delta_{S} / \bar{M}_{0, n}} \equiv\left(\pi_{1}\right)^{*}\left(-\psi_{x}\right)+\left(\pi_{2}\right)^{*}\left(-\psi_{x}\right)
$$

(cf. [KMcK, Lemma 4.5). Since the tautological classes $\psi_{x}$ are nef (cf. Kap), it follows that $\Delta_{S}$ has anti-nef normal bundle, that is, $C \cdot \Delta_{S} \leq 0$ for every irreducible curve $C \subseteq \Delta_{S}$. We shall often use certain maps between moduli spaces which we call boundary restrictions:

Definition 2. For $m, n \geq 3$ and $n_{x_{1}}, \ldots, n_{x_{m}} \geq 1$ such that $n=n_{x_{1}}+\cdots+n_{x_{m}}$, we define the map $\nu: \bar{M}_{0, m} \longrightarrow \bar{M}_{0, n}$ which takes a rational $m$-pointed curve $\left(C, x_{1}, \ldots, x_{m}\right)$ to a rational $n$-pointed curve by attaching a fixed rational $\left(n_{x_{i}}+1\right)$ pointed curve at each point $x_{i}$ for $1 \leq i \leq m$.

Note that if $n_{x_{i}}=1$ for some $i$, then this amounts to not having attached anything at $x_{i}$. Moreover, any composition of boundary restrictions will be homotopic to a single boundary restriction; in particular, they will induce the same map in homology. We make the following simple observation:

Proposition 6. Given integers $g, n$ with $g+n \geq 8$, to conclude that $\overline{N E}_{1}\left(\bar{M}_{g, n}\right)$ is generated by $F$-curves it suffices to prove that for all F-divisors $D$ on $\bar{M}_{0, g+n} / S_{g}$ and for all boundary restrictions $\nu: \bar{M}_{0, k} \rightarrow \bar{M}_{0, g+n}$, where $8 \leq k \leq g+n$, the pull-back $\nu^{*}(D)$ is a nonnegative combination of boundary divisors.

Proof. We apply GKM], Theorem 0.3. We start with an $F$-divisor $D$ on $\bar{M}_{0, g+n} /$ $S_{g}$, and we want to show that $D$ is nef. Since $D$ is a nonnegative combination of boundary divisors, we only have to show that $C \cdot D \geq 0$ for all irreducible curves in a boundary divisor $\Delta_{S} \cong \bar{M}_{0,|S|+1} \times \bar{M}_{0,\left|S^{c}\right|+1}$. By hypothesis $D_{\mid M_{0,|S|+1}}$ and $D_{\mid \bar{M}_{0,\left|S^{c}\right|+1}}$ are both effective combinations of boundary; hence we have to test the nefness of $D$ only against curves sitting in the boundary of $\bar{M}_{0,|S|+1}$ and of $\bar{M}_{0,\left|S^{c}\right|+1}$, and we can descend all the way to a moduli space $\bar{M}_{0, n}$ with $n \leq 7$. Since in this range the $F$-curves generate $\overline{N E}_{1}\left(\bar{M}_{0, n}\right)$ (cf. Proposition 5), the conclusion follows.

Let us consider a boundary restriction $\nu: \bar{M}_{0, m} \rightarrow \bar{M}_{0, n}$ given by the partition $\left(n_{x_{1}}, \ldots, n_{x_{m}}\right)$ of $n$, where we assume that $n_{x_{j}} \geq 2 \Leftrightarrow j \in\{1, \ldots, r\}$. We also denote by $A:=\left\{x_{r+1}, \ldots, x_{m}\right\}$ the set of remaining marked points; hence $n_{y}=1$ for all $y \in A$. For $2 \leq i \leq m-2$ and for $S \subset\left\{x_{1}, \ldots, x_{r}\right\}$ we define

$$
B_{i}^{S}:=\sum_{\substack{T \subset\{1, \ldots, m\},|T|=i \\ T \cap\left\{x_{1}, \ldots, x_{r}\right\}=S}} \delta_{T} .
$$

The adjunction formula for a bounday restriction $\nu: \bar{M}_{0, m} \rightarrow \bar{M}_{0, n}$ reads

$$
\nu^{*}\left(K_{\bar{M}_{0, n}}\right)=K_{\bar{M}_{0, m}}+\sum_{n_{x} \geq 2} \psi_{x} .
$$


The next statement describes the effect a boundary restriction has on homology.

Proposition 7. Let $D \equiv \sum_{i=2}^{\lfloor n / 2\rfloor} r_{i} B_{i}$ be a divisor on $\widetilde{M}_{0, n}$. If $\nu: \bar{M}_{0, m} \rightarrow \bar{M}_{0, n}$ is a boundary restriction, then

$$
\nu^{*} D \equiv \sum_{\substack{S \subset\left\{x_{1}, \ldots, x_{r}\right\} \\|S| \leq\lfloor r / 2\rfloor, i \geq|S|}} c_{i}^{S} B_{i}^{S}
$$

where

$$
c_{i}^{S}:=r_{i+\sum_{x \in S} n_{x}-|S|}-\frac{(m-i)(m-1-i)\left(\sum_{x \in S} r_{n_{x}}\right)+i(i-1)\left(\sum_{x \in S^{c}} r_{n_{x}}\right)}{(m-1)(m-2)} .
$$

Proof. We start with the case when only one $n_{x}$ is $\geq 2$. We obtain that $\nu^{*}\left(B_{i}\right)=$ $B_{i}^{\emptyset}+B_{i-n_{x}+1}^{x}-(? i) \psi_{i}$, where $(? i)=1$ if $i=n_{x}$ and 0 otherwise. By iteration, in the case when $n_{x_{j}} \geq 2$ for $1 \leq j \leq r$, we can write that

$$
\nu^{*}\left(B_{i}\right)=\sum_{S \subset\left\{x_{1}, \ldots, x_{r}\right\}} B_{i-\sum_{x \in S} n_{x}+|S|}^{S}-\sum_{\substack{x \in\left\{x_{1}, \ldots, x_{r}\right\} \\ n_{x}=i}} \psi_{x} .
$$

To read this formula correctly, when $i=n / 2$ the first sum is taken only over the subsets $S \subset\left\{x_{1}, \ldots, x_{r}\right\}$ containing $x_{1}$, that is, we do not count both $S$ and $S^{c}$. Moreover, we make the convention that $B_{j}^{S}=0$ whenever $j<|S|$ or $j \geq m-1$. Now, replacing each $\psi_{x}$ by its average formula from Lemma 1, we get the desired formula.

We have already seen that $\overline{N E}_{1}\left(\bar{M}_{g, n}\right)$ is spanned by $F$-curves whenever $g+n \leq$ 7. We now present an entirely combinatorial computation of $\overline{N E}_{1}\left(\bar{M}_{6,2}\right)$ which can serve as a model for other cases when $g+n$ is rather small.

Proposition 8. Every $F$-divisor on $\bar{M}_{0,8} / S_{6}$ is linearly equivalent to an effective combination of boundary divisors. It follows that $\overline{N E}_{1}\left(\bar{M}_{6,2}\right)$ is generated by $F$ curves.

Proof. Let us denote by $X:=\bar{M}_{0,8} / S_{6}$ and by $x, y \in\{1, \ldots, 8\}$ the marked points on which $S_{6}$ fails to act. We identify divisors on $X$ with $S_{6}$-invariant divisors on $\bar{M}_{0,8}$. A basis for $\operatorname{Pic}(X)$ is given by the following collection of divisor classes:

$$
\begin{array}{rlrl}
\delta_{x 1} & :=\sum_{a \neq x, y} \delta_{x a}, & \delta_{y 1} & :=\sum_{a \neq x, y} \delta_{y a}, \\
\delta_{x 2} & :=\sum_{a, b \in\{x, y\}^{c}} \delta_{x a b}, & :=\sum_{a, b \in\{x, y\}^{c}} \delta_{y a b}, \\
\delta_{2} & :=\sum_{a, b \in\{x, y\}^{c}} \delta_{a b}, & \sum_{a, b, c \in\{x, y\}^{c}} \delta_{a b c}, \\
\delta_{x y 1} & :=\sum_{a \neq x, y} \delta_{a x y}, & \delta_{x y 2}=\delta_{4}:=\sum_{a, b \in\{x, y\}^{c}} \delta_{x y a b}, \\
\delta_{x 3}= & \delta_{y 3}:=\sum_{a, b, c \in\{x, y\}^{c}} \delta_{x a b c} .
\end{array}
$$

There is a 10 -th $S_{6}$-invariant divisor class on $\bar{M}_{0,6}$, namely $\delta_{x y}$, which can be expressed in this basis using our average formula from Proposition 1 .

$$
\delta_{x y}=\frac{1}{6}\left(\delta_{x 1}+\delta_{y 1}\right)+\frac{4}{15}\left(\delta_{x 2}+\delta_{y 2}\right)+\frac{3}{20} \delta_{x 3}-\frac{1}{5} \delta_{3}-\frac{2}{3} \delta_{x y 1}-\frac{2}{5} \delta_{x y 2}-\frac{1}{15} \delta_{2} .
$$


We now start with an arbitrary $F$-divisor on $X$ :

$$
\begin{aligned}
D \equiv & b_{x 1} \delta_{x 1}+b_{y 1} \delta_{y 1}+b_{x 2} \delta_{x 2}+b_{y 2} \delta_{y 2}+b_{x 3} \delta_{x 3}+b_{x y 1} \delta_{x y 1} \\
& +b_{x y 2} \delta_{x y 2}+b_{2} \delta_{2}+b_{3} \delta_{3} .
\end{aligned}
$$

The coefficients of $D$ are subject to $28 F$-inequalities coming from all equivalence classes of partitions of $\{1, \ldots, 8\}$ into four subsets modulo the $S_{6}$ action. By $\left(3_{x}, 2_{y}, 2,1\right)$, for instance, we shall denote a partition of type $(3: 2: 2: 1)$ such that $x$ is contained in the subset with 3 elements and $y$ is part of one of the subsets with 2 elements. The proof that $D$ is linearly equivalent to an effective boundary consists of two parts and is similar to the proof of Theorem 2

(i) If $b_{2} \geq 0$, then all coefficients in (8) are nonnegative.

(ii) If $b_{2}<0$, then we substitute $\delta_{2}$ using (7) and we call the resulting divisor class $D_{2} \equiv D$. We then take the linear combination $D^{\prime}:=5 D+D_{2} \equiv 6 D$ and show that the resulting expression,

$$
\begin{aligned}
D^{\prime} \equiv & \left(6 b_{x 1}+\frac{5}{2} b_{2}\right) \delta_{x 1}+\left(6 b_{y 1}+\frac{5}{2} b_{2}\right) \delta_{y 1}+\left(6 b_{x 2}+4 b_{2}\right) \delta_{x 2}+\left(6 b_{y 2}+\delta_{2}\right) \delta_{y 2} \\
& +\left(6 b_{x 3}+\frac{9}{4} b_{2}\right) \delta_{x 3}+\left(6 b_{x y 1}-10 b_{2}\right) \delta_{x y 1}+\left(6 b_{x y 2}-6 b_{2}\right) \delta_{x y 2} \\
& +\left(6 b_{3}-3 b_{2}\right) \delta_{3}-15 b_{2} \delta_{x y}
\end{aligned}
$$

is effective. We present step (ii), step (i) being similar, only simpler.

We thus assume that $b_{2}<0$, and we show that all coefficients in (9) are nonnegative. We start with the $\delta_{x y 2}$ coefficient, whose nonnegativity follows from the $F$-inequality corresponding to the partition $\left(2_{x y}, 2,2,2\right)$. The fact that the $\delta_{3}$ coefficient is $\geq 0$ comes by using $\left(2_{x y}, 2,2,2\right)$ and $\left(4_{x y}, 2,1,1\right)$.

The other inequalities are slightly more involved. We first prove that $b_{x 1} \geq 0$ by combining $\left(5_{y}, 1_{x}, 1,1\right),\left(4_{y}, 2,1_{x}, 1\right)$ and $\left(3,3,1_{x}, 1_{y}\right)$. By symmetry we also obtain that $b_{y 1} \geq 0$.

Next, by adding together $\left(4_{x}, 2_{y}, 1,1\right),\left(4_{y}, 2_{x}, 1,1\right),\left(3,3,1_{x}, 1_{y}\right),\left(3_{x}, 2_{y}, 2,1\right)$ and $\left(3_{y}, 2_{x}, 2,1\right)$, we find that

$$
4\left(b_{x 2}+b_{y 2}\right)+2 b_{2} \geq 3\left(b_{x 1}+b_{y 1}\right) \geq 0,
$$

and since we have assumed $b_{2}<0$, we get that $b_{x 2}+b_{y 2} \geq 0$. Now $\left(3_{x}, 3_{y}, 1,1\right)$ gives that $2 b_{x 3}+b_{2} \geq b_{x 2}+b_{y 2} \geq 0$; thus we also have that $b_{x 3} \geq 0$. We can now prove that the remaining coefficients in (9) are nonnegative as well.

We start with the $\delta_{x 3}$ coefficient, which is nonnegative because $2 b_{x 3}+b_{2} \geq 0$ and $b_{x 3} \geq 0$. To deal with the $\delta_{x 2}$ coefficient we combine $\left(4_{y}, 2_{x}, 1,1\right),\left(5_{y}, 1_{x}, 1,1\right)$ with $b_{x 3} \geq 0$. By symmetry, the $\delta_{y 2}$ coefficient is also $\geq 0$. For the $\delta_{x 1}$ coefficient we use that $\delta_{x 1} \geq 0$ together with $\left(5_{y}, 1_{x}, 1,1\right)$. Again, by symmetry, the $\delta_{y 1}$ coefficient is also $\geq 0$.

We are left with the $\delta_{x y 1}$ coefficient, whose nonnegativity follows from $b_{2}<0$ together with $\left(4,2_{x y}, 1,1\right)$ and $\left(2_{x y}, 2,2,2\right)$. Note that we only used 10 of the 28 $F$-inequalities.

We use Proposition 8 to compute the Mori cones of $\bar{M}_{g, 1}$ when $g \leq 8$ :

Proposition 9. The cone $\overline{N E}_{1}\left(\bar{M}_{g, 1}\right)$ is generated by $F$-curves for all $g \leq 8$.

Proof. Since the case $g \leq 6$ is settled by Proposition 5 we only need to deal with $\bar{M}_{7,1}$ and $\bar{M}_{8,1}$. We only present the $g=8$ case, $g=7$ being similar. According to Proposition 6 it suffices to show that for any $F$-divisor $D$ on $\bar{M}_{0,9} / S_{8}$ we have 
that (i) D is linearly equivalent to an effective sum of boundaries, and (ii) for a boundary restriction $\nu: \bar{M}_{0,8} \rightarrow \bar{M}_{0,9}, \nu^{*}(D)$ is linearly equivalent to an effective sum of boundaries.

To prove (i) we define $Y:=\bar{M}_{0, n} / S_{n-1}$ and denote by $x$ the marked point on which $S_{n-1}$ does not act. A basis for $\operatorname{Pic}(Y)$ is given by the classes $\delta_{i}^{\{x\}, 1}$ for $i=1, \ldots, n-3$. In this case the $S_{n-1}$-invariant boundary classes on $\bar{M}_{0, n}$ are independent, which considerably reduces the combinatorial complexity of the problem. We write (uniquely) the class of any $F$-divisor $D$ on $Y$ in this basis, and the positivity of the coefficients follows in a straightforward way from the $F$ inequalities. We omit the details.

For (ii) it is enough to notice that for any boundary restriction $\nu: \bar{M}_{0,8} \rightarrow \bar{M}_{0,9}$ the pull-back $\nu^{*}(D)$ is an $F$-divisor on $\bar{M}_{0,8} / S_{6}$. So by Proposition 8 it is equivalent to an effective sum of boundary classes.

We are now going to prove that $\overline{N E}_{1}\left(\widetilde{M}_{0, n}\right)$ is spanned by $F$-curves for all $n \leq 13$. We use our Theorem 2 to give a Mori-theoretic sufficient condition for an extremal ray on $\bar{M}_{0, n}$ to be generated by an $F$-curve. The next theorem is an improvement of [KMcK], Theorem 1.2. We recall that $\Delta$ denotes the total boundary in $\bar{M}_{0, n}$.

Theorem 4. Let $R$ be an extremal ray in $\overline{N E}_{1}\left(\bar{M}_{0, n}\right)$. If there exists a nonempty effective $\mathbb{Q}$-divisor $G$ on $\bar{M}_{0, n}$ such that $\Delta-G$ is also effective and $\left(K_{\bar{M}_{0, n}}+G\right) \cdot R \leq$ 0 , then $R$ is contractible and it is spanned by an F-curve.

Proof. We follow the same lines as in [KMcK]. Let us write $G \equiv \sum_{S} a_{S} \Delta_{S}$, with $0 \leq a_{S} \leq 1$. We claim that the ray $R$ descends to some boundary divisor. Suppose this is not the case; hence $R \cdot \Delta_{S} \geq 0$ for all $S$ and $R \cdot K_{\bar{M}_{0, n}} \leq 0$. Since there exists an ample divisor on $\bar{M}_{0, n}$ having the same support as $\Delta$, namely the tautological divisor $\kappa_{1}$, we find that $R$ is generated by a contractible curve $C$. We denote by $f: \bar{M}_{0, n} \rightarrow Y$ the contraction. The curve $C$ does not come from the boundary. Hence $f_{\mid \Delta}$ is finite, and we can apply $\mathrm{KMcK}$, Proposition 2.5, to conclude that the exceptional locus $\operatorname{Exc}(f)$ is a curve. On the other hand, using the deformationtheoretic bound for the dimension of the Hilbert scheme (cf. [K], Theorem 1.14)

$$
\operatorname{dim}_{[C]} \operatorname{Hilb}\left(\bar{M}_{0, n}\right) \geq-K_{\bar{M}_{0, n}} \cdot C+n-6 \geq 1 \quad(\text { for } n \geq 7),
$$

we conclude that $C$ deforms inside $\bar{M}_{0, n}$, which contradicts that $\operatorname{Exc}(\mathrm{f})$ is a curve. This argument breaks down for $n \leq 6$, but in that case we can directly invoke Theorem $[2$ and finish the proof. Thus in any case we may assume that $R$ is contained in some boundary divisor $\Delta_{T}$, and since $\overline{N E}_{1}\left(\Delta_{T}\right)=\overline{N E}_{1}\left(\bar{M}_{0,|T|+1}\right) \times$ $\overline{N E}_{1}\left(\bar{M}_{0,\left|T^{c}\right|+1}\right)$, we may as well assume that, say, $R \subseteq \overline{N E}_{1}\left(\bar{M}_{0,|T|+1}\right)$.

If $m:=|T|+1$, we denote by $\nu: \bar{M}_{0, m} \rightarrow \bar{M}_{0, n}$ the corresponding boundary restriction and by $x$ the point of attachment of the fixed $\left(\left|T^{c}\right|+1\right)$-pointed rational curve. We replace $G$ by the effective divisor $G^{\prime}:=G+\left(1-a_{T}\right) \Delta_{T}$. The boundary $\Delta_{T}$ has anti-nef normal bundle; hence $\left(K_{\bar{M}_{0, n}}+G^{\prime}\right) \cdot R \leq 0$.

By adjunction, $\nu^{*}\left(K_{\bar{M}_{0, n}}\right)=K_{\bar{M}_{0, m}}+\psi_{x}$, while according to Proposition 7 we have that $\nu^{*}\left(G^{\prime}\right)=\tilde{G}-\psi_{x}$, where $\tilde{G}$ is an effective divisor such that $\Delta_{\bar{M}_{0, m}}-\tilde{G}$ is effective too. Thus $\left(K_{\bar{M}_{0, m}}+\tilde{G}\right) \cdot R \leq 0$, that is, we have exactly the initial situation on a lower-dimensional moduli space, and the conclusion follows inductively. 
Now we show that for $n \leq 13$ every extremal ray on $\widetilde{M}_{0, n}$ satisfies the conditions from Theorem 4 We start with an extremal ray $R \subseteq \overline{N E}_{1}\left(\widetilde{M}_{0, n}\right)$ and denote by $E$ a supporting nef divisor of $R$. Proposition $[5$ gives that $E$ is big, that is, $E \in \operatorname{int}\left(\overline{N E}^{1}\left(\bar{M}_{0, n}\right)\right)$. Since $K_{\bar{M}_{0, n}}=\sum_{j=2}^{\left\lfloor\frac{n}{2}\right\rfloor}(j(n-j) /(n-1)-2) B_{j}$, clearly $-K_{\bar{M}_{0, n}}$ is not effective for $n \geq 7$. Following Keel and McKernan, we intersect the line segment in $N S\left(\bar{M}_{0, n}\right)$ joining $-K_{\bar{M}_{0, n}}$ and $E$ with the boundary of $\overline{N E}^{1}\left(\widetilde{M}_{0, n}\right)$ to get a symmetric boundary class $\Delta_{E}$ such that $\lambda E \equiv K_{\bar{M}_{0, n}}+\Delta_{E}$ for some $\lambda>0$. We can write $\Delta_{E} \equiv \sum_{i=2}^{\left\lfloor\frac{n}{2}\right\rfloor} r_{i} B_{i}$, where $r_{i} \geq 0$ (cf. Proposition[5). That $\Delta_{E}$ is on an extremal face of the cone means $r_{i}=0$ for at least one $i$ with $2 \leq i \leq\lfloor n / 2\rfloor$. If we can prove that $r_{i} \leq 1$ for all $i$, then Theorem 4 gives that $R$ is generated by an $F$-curve.

To achieve this we write out all $F$-inequalities for the nef divisor $K_{\bar{M}_{0, n}}+\Delta_{E}$ : We define the function $f(a, b, c, d)$ to be 2 minus the number of variables equal to 1. For any partition $(a, b, c, d)$ of $n$ into positive integers we consider the associated $F$-curve given by a boundary restriction $\nu: \bar{M}_{0,4} \rightarrow \bar{M}_{0, n}$. Then, using (6),

$$
\begin{aligned}
& \left(K_{\bar{M}_{0, n}}+\Delta_{E}\right) \cdot \nu\left(\bar{M}_{0,4}\right) \\
& \quad=f(a, b, c, d)+r_{a+b}+r_{a+c}+r_{a+d}-r_{a}-r_{b}-r_{c}-r_{d} \geq 0 .
\end{aligned}
$$

Theorem 5. For $n \leq 13$ any nontrivial nef divisor on $\widetilde{M}_{0, n}$ is of the form $K_{\bar{M}_{0, n}}+$ $\Delta_{E}$, with $0 \leq \Delta_{E} \leq \Delta$. It follows that $\overline{N E}_{1}\left(\bar{M}_{g}\right)$ is generated by $F$-curves for all $g \leq 13$.

Proof. We start with the nef divisor $K_{\bar{M}_{0, n}}+\Delta_{E}$, where $\Delta_{E} \equiv \sum_{j=2}^{\lfloor n / 2\rfloor} r_{j} B_{j}$, where $r_{j} \geq 0$ for all $j$ and there is $2 \leq i \leq\lfloor n / 2\rfloor$ such that $r_{i}=0$. By using all $F$ inequalities that the coefficients $r_{j}$ are subject to, we conclude that $r_{j} \leq 1$. We carry this out only for $n=13$, the case $n=12$ being entirely similar. We list all $F$-inequalities for $n=13$ :
(1) $3 r_{2} \geq r_{3}+1$,
(2) $2 r_{3} \geq r_{4}$
(3) $r_{2}+2 r_{4} \geq r_{3}+r_{5}$,
(4) $r_{2}+2 r_{5} \geq r_{4}+r_{6}$
(5) $r_{2}+r_{6} \geq r_{5}$
(6) $1+2 r_{3}+r_{4} \geq 2 r_{2}+r_{5}$,
(7) $1+r_{4}+r_{5} \geq r_{2}+r_{6}$,
(8) $1+r_{3}+r_{5} \geq r_{2}+r_{4}$
(9) $1+r_{3}+$
(10) $1+2 r_{4} \geq 2 r_{3}$,
(11) $1+r_{6} \geq r_{3}$
(12) $1+3 r_{5} \geq 3 r_{4}$
(13) $2+3 r_{4} \geq 3 r_{2}+r_{6}$,
(14) $2+r_{4}+2 r_{5} \geq 2 r_{2}+r_{3}+r_{6}$, (15) $2+2 r_{6} \geq 2 r_{2}+r_{5}$,
(16) $2+r_{5}+r_{6} \geq r_{2}+2 r_{3}$, (17) $2+r_{5}+2 r_{6} \geq r_{2}+r_{3}+2 r_{4}$,

and finally (18) $2+3 r_{6} \geq 3 r_{3}+r_{4}$.

From (1) we see that $r_{2}>0$. We have four cases:

(i) $r_{3}=0$. Then from (2) we have $r_{4}=0$, while from (6) we get $r_{5}<1$ and $r_{2} \leq 1 / 2$. Assume now that $r_{6} \geq 1$. Then (3) and (7) combined give $r_{5}=r_{2}$ and $r_{6}=1$. From (13) we get that $r_{2} \leq 1 / 3$, while (4) gives that $r_{2} \geq 1 / 3$, hence $r_{2}=r_{5}=1 / 3$. Thus either $r_{j}<1$ for all $j$, or else $\Delta_{E} \equiv \frac{1}{3}\left(B_{2}+B_{5}\right)+B_{6}$.

(ii) $r_{4}=0$. Use (10), (13) and (3) to get that $r_{3} \leq 1 / 2, r_{2} \leq 2 / 3$ and $r_{5} \leq 2 / 3$. We assume again that $r_{6} \geq 1$. Then (3) and (7) are compatible only when $r_{2}=r_{5}$ and $r_{3}=0$, that is, we are back to case (i).

(iii) $r_{5}=0$. Inequality (12) yields $r_{4} \leq 1 / 3$, while from (13) we get that $r_{2} \leq 1$. Moreover, if $r_{2}=1$, then $r_{6}=0, r_{4}=1 / 3$ and $r_{3}=1 / 3$. So in this way we get our 
second exceptional case, $\Delta_{E} \equiv B_{2}+\frac{1}{3}\left(B_{3}+B_{4}\right)$. On the other hand, if $r_{2}<1$, then from (4) we have that $r_{6}<1$, and finally from (10) we obtain that $r_{3} \leq 5 / 6$. (iv) $r_{6}=0$. From (18) we have that $r_{3} \leq 2 / 3$, while (5) and (15) give that $r_{5} \leq 2 / 3$ and $r_{2} \leq 1$. Moreover, $r_{2}=1$ implies $r_{5}=0$; so we are back to case (iii). Then we use (12), which gives $r_{4} \leq 1$. If $r_{4}=1$, from (18) we have $r_{3} \leq 1 / 3$, while from (2) we have $r_{3} \geq 1 / 2$, a contradiction, and so this last case does not occur.

Remark. For $n \leq 11$ every nontrivial $F$-class on $\widetilde{M}_{0, n}$ is of the form $K_{\bar{M}_{0, n}}+\Delta_{E}$, where $\Delta_{E}$ is a pure boundary, that is, $\Delta_{E}=\sum_{i} r_{i} B_{i}$ where $0 \leq r_{i}<1$ (cf. [KMcK], Corollary 5.3). The previous proof shows that on $\widetilde{M}_{0,13}$ there are exactly two $F$ classes not of this form:

$$
K_{\bar{M}_{0,13}}+\frac{1}{3}\left(B_{2}+B_{5}\right)+B_{6} \quad \text { and } \quad K_{\bar{M}_{0,13}}+B_{2}+\frac{1}{3}\left(B_{3}+B_{4}\right) .
$$

On $\widetilde{M}_{0,12}$ there is just one such class, namely $K_{\bar{M}_{0,12}}+\left(B_{2}+B_{5}\right) / 3+B_{6}$. For $n \geq 14$ it is no longer true that any nontrivial $F$-divisor on $\widetilde{M}_{0, n}$ is numerically equivalent to $K_{\bar{M}_{0, n}}+\Delta_{E}$, where $\Delta_{E}=\sum_{i} r_{i} B_{i}$ with $0 \leq r_{i} \leq 1$. When $n=14$, for instance, the $F$-class

$$
K_{\bar{M}_{0,14}}+\frac{1}{3}\left(B_{2}+B_{5}\right)+B_{6}+r_{7} B_{7},
$$

where $r_{7} \in[1,4 / 3]$, is not of this form.

\section{REFERENCES}

[AC] E. Arbarello, M. Cornalba, Calculating cohomology groups of moduli spaces of curves via algebraic geometry, Inst. Hautes Études Sci. Publ. Math. No. 88(1998), 97-127. MR 2001h:14030

[Fa1] C. Faber, Intersection-theoretical computations on $\bar{M}_{g}$, Parameter Spaces (Warsaw 1994), 71-81, Banach Center Publ. 36, 1996. MR 98j:14033

[Fa2] C. Faber, The nef cone of $\bar{M}_{0,6}$ : a proof by inequalities only, preprint.

[G] A. Gibney, Fibrations of $\bar{M}_{g, n}$, Ph.D. Thesis, University of Texas, 2000.

[GKM] A. Gibney, S. Keel, I. Morrison, Towards the ample cone of $\bar{M}_{g, n}$, J. Amer. Math. Soc. 15(2002), 273-294.

[HT] B. Hassett, Y. Tschinkel, On the effective cone of the moduli space of pointed rational curves, math.AG/0110231.

[H] B. Hunt, The geometry of some special arithmetic quotients, Lecture Notes in Mathematics 1637, Springer 1996. MR 98c:14033

[HMo] J. Harris, I. Morrison, Moduli of curves, Springer, 1998. MR 99g:14031

[Kap] M. Kapranov, Veronese curves and the Grothendieck-Knudsen moduli space $\bar{M}_{0, n}$, J. of Algebraic Geometry, 2 (1993), 239-262. MR 94a:14024

[Ke] S. Keel, Intersection theory on moduli spaces of n-pointed curves of genus zero, Trans. Amer. Math. Soc. 330(1992), 545-574. MR 92f:14003

[KMcK] S. Keel, J. McKernan, Contractible extremal rays on $\bar{M}_{0, n}$, math.AG/9607009.

[K] J. Kollár, Rational curves on algebraic varieties, Springer 1996. MR 98c:14001

[Ve] P. Vermeire, A counterexample to Fulton's conjecture on $\bar{M}_{0, n}$, J. of Algebra $248(2002)$, $780-784$.

Department of Mathematics, University of Michigan, Ann Arbor, Michigan 481091109

E-mail address: gfarkas@umich.edu

Department of Mathematics, University of Michigan, Ann Arbor, Michigan 481091109

E-mail address: agibney@umich.edu 